\title{
Novel Hydrogen Clathrate Hydrate
}

Yu Wang, ${ }^{1}$ Konstantin Glazyrin, ${ }^{2}$ Valery Roizen, ${ }^{3}$ Artem R. Oganov, ${ }^{4}$ Ivan Chernyshov $\odot,{ }^{5}$ Xiao Zhang, ${ }^{1}$ Eran Greenberg, ${ }^{6, *}$

Vitali B. Prakapenka $\odot,{ }^{6}$ Xue Yang, ${ }^{1}$ Shu-qing Jiang, ${ }^{1}$ and Alexander F. Goncharov $\circledast^{1,7}$

${ }^{1}$ Key Laboratory of Materials Physics, Institute of Solid State Physics, HFIPS, Chinese Academy of Sciences, Hefei 230031, Anhui, People's Republic of China

${ }^{2}$ Photon Science, Deutsches Elektronen-Synchrotron, Notkestrasse 85, 22607 Hamburg, Germany

${ }^{3}$ Moscow Institute of Physics and Technology (State University), Dolgoprudnyi, Moscow region, 141701 Russia

${ }^{4}$ Skolkovo Institute of Science and Technology, Skolkovo, 14302 Russia

${ }^{5}$ TheoMAT Group, ChemBio Cluster, ITMO University, Lomonosova 9, St. Petersburg, 191002 Russia

${ }^{6}$ Center for Advanced Radiations Sources, University of Chicago, Chicago, Illinois 60637, USA

${ }^{7}$ Earth and Planets Laboratory, Carnegie Institution of Washington, 5251 Broad Branch Road NW, Washington, D.C. 20015, USA

(Received 12 July 2020; accepted 20 November 2020; published 18 December 2020)

\begin{abstract}
We report a new hydrogen clathrate hydrate synthesized at $1.2 \mathrm{GPa}$ and $298 \mathrm{~K}$ documented by singlecrystal x-ray diffraction, Raman spectroscopy, and first-principles calculations. The oxygen sublattice of the new clathrate hydrate matches that of ice II, while hydrogen molecules are in the ring cavities, which results in the trigonal $\boldsymbol{R} 3 \boldsymbol{c}$ or $\boldsymbol{R} \overline{\boldsymbol{3}} \boldsymbol{c}$ space group (proton ordered or disordered, respectively) and the composition of $\left(\mathrm{H}_{2} \mathrm{O}\right)_{6} \mathrm{H}_{2}$. Raman spectroscopy and theoretical calculations reveal a hydrogen disordered nature of the new phase $C_{1}^{\prime}$, distinct from the well-known ordered $C_{1}$ clathrate, to which this new structure transforms upon compression and/or cooling. This new clathrate phase can be viewed as a realization of a disordered ice II, unobserved before, in contrast to all other ordered ice structures.
\end{abstract}

DOI: $10.1103 /$ PhysRevLett.125.255702

Water molecules in solid states tend to form hydrogenbonded 3D frameworks. There are many possibilities for water molecules to associate giving rise to a great variety of configurations and structures observed in nature and at artificial extreme conditions. Pure water is famous for crystallizing in ices of different structures even at moderate pressures resulting in a very complex phase diagram below 2 GPa (e.g., Refs. [1,2] and references therein). This structural diversity is also extended for different water-bearing compounds. Indeed, a variety of clathrate hydrates is known, with water molecules arranging themselves to form polyhedral frameworks with cavities containing foreign guest molecules. The latter interact only weakly with water cages via van der Waals forces. Gas hydrates can be found in many natural environments such as, for example, deep sea sediments and they can play an important role in the planetary formation and evolution [3,4].

Hydrogen clathrate hydrates have been recently thoroughly investigated at elevated pressures. These materials are of interest for hydrogen storage and are expected to occur in natural environments, icy satellites and comets being the most prominent examples. There are four major crystal structures, which these materials commonly form depending on pressure-temperature $(P-T)$ conditions, and they differ in structure and composition (Fig. 1). The volumes of cavities in these materials decrease with compression, and the high-pressure structures are

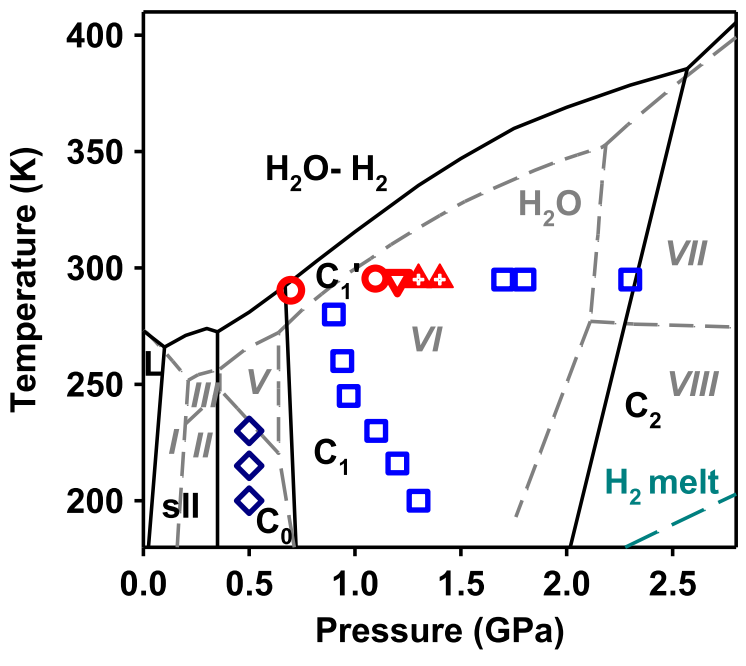

FIG. 1. Tentative $P-T$ phase diagram for $\mathrm{H}_{2}-\mathrm{H}_{2} \mathrm{O}$ compounds superimposed on those of pure $\mathrm{H}_{2} \mathrm{O}$ and $\mathrm{H}_{2}$. Black solid lines are the $\mathrm{H}_{2}-\mathrm{H}_{2} \mathrm{O}$ phase equilibrium lines proposed in Ref. [9], while gray dashed lines correspond to phase lines for pure water (e.g., Ref. [18]) and dark cyan dashed line is for pure $\mathrm{H}_{2}$ [19]. Dark blue open diamonds represent experimentally determined $P-T$ conditions in our work for the $C_{0}$ phase. A new $C_{1}^{\prime}$ phase (open red circles and red triangles) appears upon pressure release of the $C_{1}$ phase at $295 \mathrm{~K}$ (open red triangle down) and on pressure increase from fluid (crossed filled red triangles up). The new $C_{1}^{\prime}$ clathrate transforms to the known $C_{1}$ phase (open blue squares) on pressure increase at $295 \mathrm{~K}$ and upon cooling down with a simultaneous pressure increase. 
commonly described as filled ices-inclusion compounds with structures closely related to known ice structures. The lowest pressure clathrate has a classical sII structure, which forms cages of two different dimensions occupied by a different number of hydrogen molecules [5]. Initially sII structure formation was inferred from the differential thermal analysis data [6], which later were confirmed via in situ optical spectroscopy and neutron diffraction investigations [5,7]. Remarkably, above $\sim 0.35 \mathrm{GPa}$ another hydrogen hydrate phase $C_{0}$ appears [8-11] consisting of interpenetrating chiral chains of hydrogen-bonded water molecules surrounding orientationally disordered hydrogen molecules in the channels aligned along the $c$ axis. This structure bears a topological similarity with the mineral quartz and is best described as a filled ice. Above 1.5 GPa, two other hydrogen hydrate phases have been found: $C_{1}$ and $C_{2}$ [12]. The structures of these materials resemble those of ices II and VII, respectively $[9,12,13]$, so they can be considered as filled ices. It is remarkable that ice II remains the only pure ice modification, which does not have a disordered counterpart $[14,15]$. On the other hand, an orientationally disordered $C_{2}$ filled ice structure was found to be stable above $3 \mathrm{GPa}$ up to at least $80 \mathrm{GPa}$, although a distortion was reported above $20 \mathrm{GPa}$ [16]. Because of experimental difficulties in assessing structure and chemical composition, especially for hydrogen atoms, an application of first-principles theory is of great benefit in these studies. Recent first-principles calculations confirmed some of the experimental structures and proposed several new ones, including a high-pressure one $\left(C_{3}\right)$ with a very large hydrogen content $\left(2: 1 \mathrm{H}_{2}: \mathrm{H}_{2} \mathrm{O}\right)$ above $38 \mathrm{GPa}$ [17].

In this Letter, we report a new hydrogen hydrate (named $C_{1}^{\prime}$ here) with a structure, which is very similar to that of $C_{1}$, but with a disordered hydrogen subsystem within the molecular water framework. This material can be synthesized at about $1.5 \mathrm{GPa}$ at room temperature. We found two possible paths of synthesis: on pressure release of a common $C_{1}$ clathrate or upon a repeated compression cycle of fluid $\mathrm{H}_{2} \mathrm{O}-\mathrm{H}_{2}$. This finding emphasizes a tremendous complexity of the structure-composition-pressure phase relationships of hydrogen hydrates and suggests an expansion of the known phase diagram with the implication to hydrogen storage and possible new hydrates in natural environments such as satellites.

The formation of clathrate hydrates is clearly recognized from Raman spectroscopy data, which reveal the incorporation of $\mathrm{H}_{2}$ molecules via the observation of the roton and vibron mode(s) (Figs. S1, S2, see Supplemental Material [20]), while characteristic lattice vibration modes indicate the clathrate compound formation and can be used for its identification. At room temperature, we observed the formation of $C_{1}$ from water-hydrogen fluid mixture upon compression at slightly higher pressures [up to 1.8(1) GPa, e.g., compared to that reported previously (e.g., Ref. [12]) most likely due to a higher compression rate. The lattice
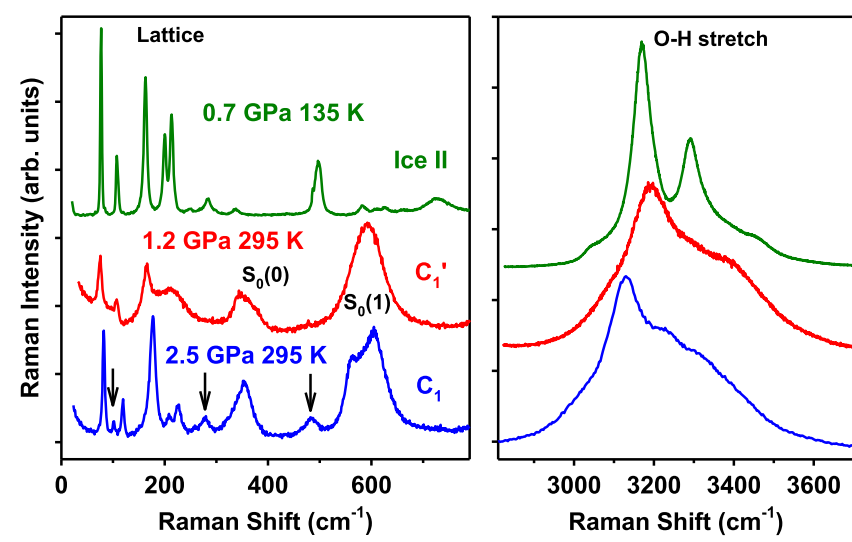

FIG. 2. Raman spectra of hydrogen hydrates $C_{1}$ and $C_{1}^{\prime}$ and ice II. The roton modes of molecular $\mathrm{H}_{2}$ are labeled; the vibron mode at about $4200 \mathrm{~cm}^{-1}$ (not shown here) is present for $C_{1}$ and $C_{1}^{\prime}$ and is absent for ice II (Figs. S1, S2). The arrows mark the unique lattice modes of the $C_{1}$ phase, which are not observed in the $C_{1}^{\prime}$ phase, while most of them have counterparts in ice II. Note the broadening of the lattice modes of $C_{1}^{\prime}$ compared to $C_{1}$. The spectra are shifted vertically for clarity. The vertical scale is different for the left (lattice vibrations) and the right $(\mathrm{O}-\mathrm{H}$ stretching modes) panels.

modes of $C_{1}$ clathrate are in agreement with the previously reported [10], however, we have extended these measurements below $100 \mathrm{~cm}^{-1}$ and observed additional peaks down to $70 \mathrm{~cm}^{-1}$ (Fig. 2). A new form of the water hydrogen compound $\left(C_{1}^{\prime}\right)$ occurs upon decompression of the $C_{1}$ clathrate down to 1.2(1) GPa at room temperature, which can be clearly recognized in the low-frequency range of the lattice vibrations (Fig. S1), where the peaks of $C_{l}$ phase broaden and some of them disappear (Fig. 2). Despite these distinctions, the lattice modes of $C_{1}, C_{1}^{\prime}$, and ice II are all alike suggesting a similar structure of $\mathrm{H}_{2} \mathrm{O}$ rings. The transition is reversible by exerting pressure on $C_{1}^{\prime}$ phase (Fig. 1). The new $C_{1}^{\prime}$ phase also crystallizes upon a repeated compression of a mixed $\mathrm{H}_{2}+\mathrm{H}_{2} \mathrm{O}$ fluid phase, which occurs on unloading of $C_{1}^{\prime}$ phase (Fig. S1). Upon cooling to below $280 \mathrm{~K}$, the $C_{1}^{\prime}$ phase transforms into $C_{1}$ as confirmed by a change in Raman signal (Fig. S2). Although these observations do not prove thermodynamic stability of $C_{1}^{\prime}$ phase, a narrow stability field cannot be ruled out.

Single-crystal x-ray diffraction (SCXRD) experiments (Fig. 3) were performed on carefully grown (see Materials and Methods and Fig. S3 in Supplemental Material [20]) and Raman characterized crystals of the $C_{1}^{\prime}$ phase, prepared for structure determination. Such measurements have been carried out by performing a collection of XRD patterns while the sample is rotated along the $\omega$ axis (perpendicular to the compression axis) across the aperture of a diamond anvil cell. During the collection, a series of XRD patterns were acquired for a set of selected $\omega$ angles by continuously scanning within $0.5^{\circ}$ each $0.5^{\circ}$ in a range of $\pm 35^{\circ}$. 


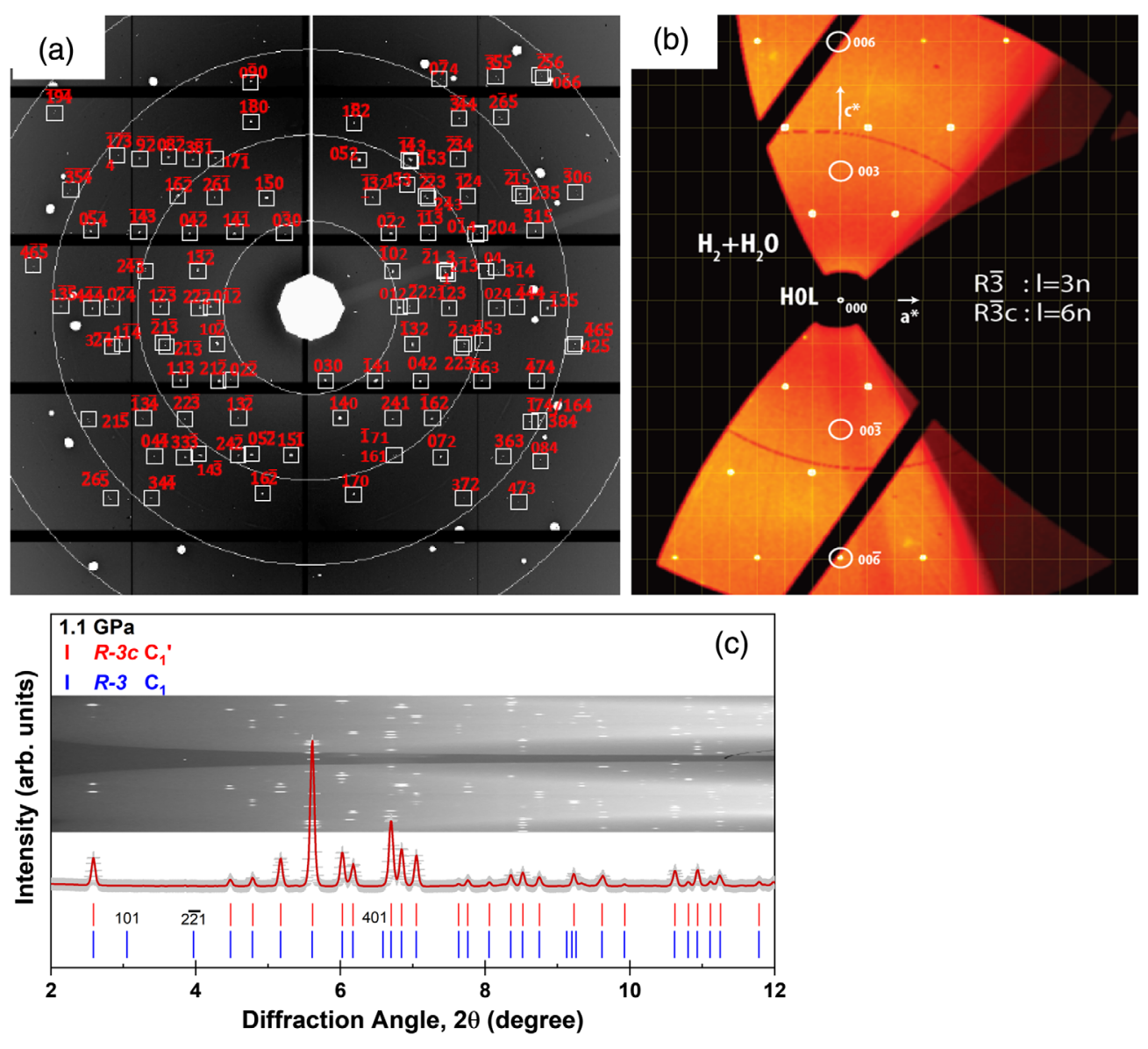

FIG. 3. (a) SCXRD 2D image collected from a single crystal of $C_{1}^{\prime}$ phase at $1.3 \mathrm{GPa}$ upon rotation of $\pm 35^{\circ}$ of the diamond anvil cell demonstrating high quality of a single crystal (Fig. S3); squares mark reflections with the given $h k l$ indices; strong unlabeled reflections are from diamond; weak unlabeled peaks could originate from other smaller single crystal(s), which could not be refined but their contribution to the intensities collected from the large crystal can be definitively singled out by a variety of algorithms (see Supplemental Material [20]); (b) $H 0 L$ slice of reciprocal space visualized by CrysAlispro software package [39] with attributed diffraction spots. Note the apparent absence of $(003)_{h k l}$ equivalent reflections; (c) Powder XRD data (the X-ray wavelength is $0.291 \AA$ ) integrated in $1 D$ pattern (gray plus symbols present the data and the red solid line is the Le Bail fit), the short red ticks correspond to $\boldsymbol{R} \overline{3} \boldsymbol{c}$ space group with lattice parameters $a=13.102(4) \AA, b=6.263(7) \AA$. The blue ticks also represent XRD of the $C_{1} \boldsymbol{R} \overline{3}$ structure (the same space group as ice II) with the same lattice parameters. Inset shows an image of the $2 \mathrm{D}$ powder XRD data of the $C_{1}^{\prime}$ phase.

Structural information on the $C_{1}^{\prime}$ phase was obtained from indexing the collected single-crystal data set [Fig. 3(a)] and the subsequent structure solution (Table I). Analyzing 1D powder XRD patterns [Fig. 3(c)] is not sufficient to distinguish this phase from $C_{1}$ and ice II with the same lattice parameters. Indeed, our powder XRD measurements were not able to detect the $C_{1}^{\prime}$ to $C_{1}$ phase transition upon pressure increase at $295 \mathrm{~K}$ (Fig. S4) because the difference is very subtle, making our single-crystal results on $C_{1}^{\prime}$ critical for identification of this phase. Ice II, which is stable below 0.7(1) GPa (Fig. 1), has very different unit cell parameters and density than $C_{1}^{\prime}$ and $C_{1}$ phases (Fig. S5), so it can be ruled out.

Indexing of the observed diffraction Bragg spots of $C_{1}^{\prime}$ phase suggested the trigonal symmetry. By analyzing the reflection rules for the observed diffraction spots, we can clearly rule out the rhombohedral $\boldsymbol{R} \overline{3}$ structure of ice II as illustrated in Fig. 3(b), which represents a slice of the reciprocal space for the $C_{1}^{\prime}$ crystal. Here, we report that $(003)_{h k l}$ as well as its equivalent reflection $(00 \overline{3})_{h k l}$ are evidently absent, while $(006)_{h k l}$ is indeed present. This observation allowed us to exclude $R 3, R 3 m, R \overline{3} m$ in a similar fashion and select $\boldsymbol{R} 3 \boldsymbol{c}$ and $\boldsymbol{R} \overline{\boldsymbol{c}} \boldsymbol{c}$ space groups as the most probable candidates. This result also shows a clear distinction between the structure of the new $C_{1}^{\prime}$ clathrate in comparison to that of $C_{1}$, which has the same $\boldsymbol{R} \overline{3}$ symmetry as ice II [12].

The individual Bragg peak intensities were extracted by means of Rigaku CrysAlisPro software [39] and analyzed by means of Olex2 [40] (with SHELX [41] backend) and additionally with Jana2006 [42]. During the structure solution, the oxygen thermal displacement parameters were refined as anisotropic $\left(U_{\text {anis }}\right)$, while isotropic approximation was used for hydrogen atoms $\left(U_{\text {iso }}\right)$. Electronic density 
TABLE I. Lattice parameters and refinement indicators of the experimental single-crystal XRD measurements at 1.3(1) GPa (see Supplemental Material [20] for the full set of data including the atomic positions) ${ }^{\mathrm{a}}$.

\begin{tabular}{|c|c|c|c|c|c|c|c|}
\hline \multirow[t]{2}{*}{ Space group } & \multirow{2}{*}{$\begin{array}{l}\text { Lattice parameters } \\
\qquad a, c(\AA)\end{array}$} & \multirow{2}{*}{$\begin{array}{l}\text { Chemical } \\
\text { formula }\end{array}$} & \multirow[t]{2}{*}{$N_{\text {obs }} / N_{\text {par }}$} & \multirow{2}{*}{$\begin{array}{l}R_{F 2} \\
\text { factor }\end{array}$} & \multirow[t]{2}{*}{ Obs. $H K L$} & \multicolumn{2}{|c|}{ Residual electronic density ${ }^{b}$} \\
\hline & & & & & & Neg. & Pos. \\
\hline $\begin{array}{l}\boldsymbol{R} \overline{3} \boldsymbol{c}, \text { No. } 167 \\
\boldsymbol{R} 3 \boldsymbol{c}, \text { No. } 161\end{array}$ & $\begin{array}{l}12.984(4), 6.243(8) \\
12.984(4), 6.243(8)\end{array}$ & $\begin{array}{l}\left(\mathrm{H}_{2} \mathrm{O}\right)_{36}\left(\mathrm{H}_{2}\right)_{6} \\
\left(\mathrm{H}_{2} \mathrm{O}\right)_{36}\left(\mathrm{H}_{2}\right)_{6}\end{array}$ & $\begin{array}{l}130 / 23 \\
130 / 33\end{array}$ & $\begin{array}{l}2.5 \% \\
2.0 \%\end{array}$ & $\begin{array}{c}1<H<17 \\
-6<K<0 \\
-7<L<8\end{array}$ & -0.1 & +0.1 \\
\hline
\end{tabular}

${ }^{\mathrm{a}} N_{\text {obs }}$, number of observed reflections with $I>3 \operatorname{sig}(I)$, where $I$ is the intensity of a Bragg spot, and sig $(I)$ is equal to the square root of intensity. $N_{\text {par }}$, number of refinement parameters. $R_{F 2}$, crystallographic $R$ factor obtained on refinement using intensities, or structure factor squared.

${ }^{\mathrm{b}}$ As reported by SHELXT, part of SHELX package.

corresponding to $\mathrm{H}_{2}$ was located in the center of the framework cavities, but due to the limited resolution and averaging property of the XRD signal, we could not resolve the position of individual $\mathrm{H}$ atoms and the assigned $\mathrm{H}_{2}$ molecule to a single position. The positions of the hydrogen atoms bound to the oxygen rings were found and refined in our analysis.

As shown in the Table I, structure solutions within the space groups $\boldsymbol{R} 3 \boldsymbol{c}$ and $\boldsymbol{R} \overline{3} \boldsymbol{c}$ describe the experimental results almost equally well. In both of these structures, linear arrays of $\mathrm{H}_{2}$ molecules are confined in channels made of the $\mathrm{H}_{2} \mathrm{O}$ rings. The difference between these two structural solutions is very subtle because of the weak scattering contribution of the hydrogen atoms. While the positions of the oxygen atoms are well constrained, there is an important difference between the solutions concerning the positions and populations of the hydrogen atom sites. Depending on the solution, the hydrogen atoms of the $\mathrm{H}_{2} \mathrm{O}$ rings are ordered for $\boldsymbol{R} 3 \boldsymbol{c}$ and disordered for $\boldsymbol{R} \overline{\boldsymbol{c}} \boldsymbol{c}$ space groups. A simple addition of a symmetry element will break the spatial confinement of $\mathrm{H}$ atoms with respect to the individual $\mathrm{O}$ atoms (as attributed to $\boldsymbol{R} 3 \boldsymbol{c}$ ) and allow a random disorder of individual $\mathrm{H}$ atoms between the equivalent general crystallographic positions of $\boldsymbol{R} \overline{\boldsymbol{z}} \boldsymbol{c}$. These half-populated crystallographic positions are lying close to the lines between the $\mathrm{O}-\mathrm{O}$ atoms forming the rings or between the oxygen positions in the adjacent rings shifted along the $c$ axis (Fig. 5). The detailed crystallographic description of our structural $\boldsymbol{R} 3 \boldsymbol{c}$ and $\boldsymbol{R} \overline{3} \boldsymbol{c}$ solutions along with the experimental and fitted reflection intensities is presented in the Supplemental Material [20] in the form of the standard structural files (CIF).

Distinguishing between the $\boldsymbol{R} 3 \boldsymbol{c}$ and $\boldsymbol{R} \overline{3} \boldsymbol{c}$ within the given XRD dataset is not straightforward, due to the limitations imposed by the high-pressure environment. Considering the data from the statistics point of view (Table I), we see that the $\boldsymbol{R} 3 \boldsymbol{c} R$ factor is not significantly lower than the one of $\boldsymbol{R} \overline{\boldsymbol{z}} \boldsymbol{c}$, although the number of fit parameters has increased by $\sim 40 \%$. This could indicate that the assignment to $\boldsymbol{R} \overline{\boldsymbol{c}} \boldsymbol{c}$ is indeed the appropriate one [43].
Additional arguments for the disordered nature of $C_{1}^{\prime}$ phase can be provided by Raman spectroscopy and first-principles calculations.

Group theory analysis of Raman modes reveals a different activity for the ordered and disordered candidate structures of $C_{1}^{\prime}$ (Table S1). This is because a disordered $\boldsymbol{R} \overline{3} \boldsymbol{c}$ structure has only one distinct oxygen position and it is centrosymmetric, making only the even symmetry (gerade) modes Raman active. Raman active modes of the $C_{1}$ structure are also subjected to this rule, but, nevertheless, this structure shows more Raman activity compared to the $\boldsymbol{R} \overline{3} \boldsymbol{c} C_{1}^{\prime}$ because it is less symmetrical. Our experiment shows less Raman activity of $C_{1}^{\prime}$ compared to $C_{1}$, thus clearly supporting a disordered $\boldsymbol{R} \overline{3} \boldsymbol{c} C_{1}^{\prime}$. In addition, a broadening of the $C_{1}^{\prime}$ Raman peaks (Fig. 2) indicates its disordered structure.

To give an independent insight into the structure and composition of $C_{1}^{\prime}$ phase, we performed the state of the art $a b$ initio calculations mainly focusing on the candidate structures $\boldsymbol{R} 3 \boldsymbol{c}$ and $\boldsymbol{R} \overline{\mathbf{z}} \boldsymbol{c}$. We examined the stability of the newly synthesized $C_{1}^{\prime}$ phase using the first-principles DFT calculations. First, we performed structure search (employing USPEX [22-24]) at the pressure of $1.4 \mathrm{GPa}$. The structure search by the USPEX algorithm predicted an orientationally ordered approximant structure with a $\boldsymbol{R} 3 \boldsymbol{c}$ space group (see Table S2). The transition pressure from the ordered approximation of $C_{1}^{\prime}$ to $C_{1}$ clathrate structure is predicted to be $\sim 2.1 \mathrm{GPa}$ (see Fig. S6), which is broadly consistent with the experiment.

Our next step was to investigate a structural disorder of hydrogen atoms, which was conducted by analyzing all $\mathrm{O}(\mathrm{H}) \cdots \mathrm{O}$ contacts. These appear to be split into two groups: "cyclic" inside $\left(\mathrm{H}_{2} \mathrm{O}\right)_{6}$ rings and "acyclic" between the rings bounding them together (Fig. 4). The randomly generated disordered structures that include the disorder of both kinds and their mixture were relaxed by VASP and their enthalpies were calculated (see Materials and Methods and Figs. S6-S7 in Supplemental Material [20]). The computations show that these disorders become energetically favorable at different temperatures: cyclic at $286 \mathrm{~K}$, acyclic 

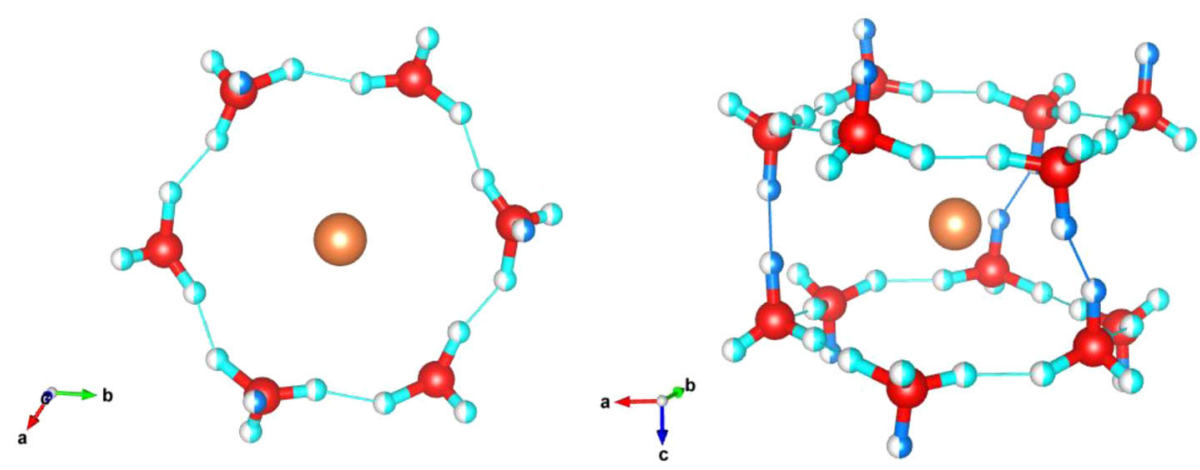

FIG. 4. The structure of $C_{1}^{\prime}$ clathrate in $\boldsymbol{R} \overline{3} \boldsymbol{c}$ setting, where the major structural motif $\left(\mathrm{H}_{2} \mathrm{O}\right.$ rings $)$ is shown in different projections to demonstrate two different disordering schemes. Orange spheres correspond to the centers of mass of the hydrogen molecules. Bluish spheres of different shades correspond to partially occupied positions for hydrogen atoms in the water cages. The solid thin light cyan and blue lines connect the duplicated hydrogen positions, generating "cyclic" (in an armchairlike virtual plane formed by six oxygen atoms) and "acyclic" (outside the plane) types of disorder, respectively.

at $68 \mathrm{~K}$, and the combination of them at $287 \mathrm{~K}$, which is again broadly in agreement with the experiment suggesting the full proton disorder in the new clathrate structure. The structure of $C_{1}^{\prime}$ phase that includes both types of disorder suggests the occupancy of hydrogen positions at the rings to be 0.5 , which agrees well with the experiments (structure $\boldsymbol{R} \overline{3} \boldsymbol{c}$ of the Table I and Table S2).

Our Raman data of $C_{1}^{\prime}$ phase strongly point to a spatially disordered $\boldsymbol{R} \overline{3} \boldsymbol{c}$ structure of the $\mathrm{H}_{2} \mathrm{O}$ rings because both the lattice and $\mathrm{OH}$-stretch bands are broader and a number of lattice modes are missing if compared to those of the ordered $C_{1}$ phase (Fig. 2, Table S1). On the other hand, the most prominent lattice mode peaks at 90 and $160 \mathrm{~cm}^{-1}$ nearly coincide in frequency for $C_{1}$ and $C_{1}^{\prime}$ phases indicating the proximity of their structures. The comparison of the experimental and theoretical (for the ordered structure) Raman spectra shows a good correspondence (Fig. S8) in a number and the spectral positions of the peaks. The lattice modes are slightly lower in frequency than the theoretically computed, and the peak broadening caused by anharmonicity or disorder varies in experiment for different peaks, while it was assumed constant in calculations. The discrepancy in the position of the $\mathrm{O}-\mathrm{H}$ stretching modes for the $C_{1}^{\prime}$ phase is at least partially due to the sensitivity of these modes to the unit cell volume (or pressure) (see Table S3).

The chemical composition of the new $C_{1}^{\prime}$ clathrate is the same as of the $C_{1}$ clathrate determined by combining SCXRD structural solution and DFT calculations. This results in the $\left(\mathrm{H}_{2} \mathrm{O}\right)_{6} \mathrm{H}_{2}$ composition. At $1.3 \mathrm{GPa}$ the density is $1.20(1) \mathrm{g} / \mathrm{cm}^{3}$. This can be compared to the density calculated from the molecular volumes of $23.6 \AA^{3} / \mathrm{H}_{2} \mathrm{O}$ for ice II [36] and $h c p$ phase I hydrogen [38] with $3.1 \AA^{3}$ per hydrogen atom (Fig. S5). The compound is some $5.5 \%$ denser than the mechanical mixture of the latter ice and hydrogen, which points to an exceptional stability of clathrate hydrates at these $P-T$ conditions, which is further emphasized by their high melting temperature, which is higher than in pure ice and hydrogen (Fig. 1). The $C_{1}^{\prime}$ clathrate can be considered as a disordered $C_{1}$, with respect to which it is likely stable given the conditions of its formation.

In conclusion, our combined SCXRD, Raman spectroscopy, and first-principles calculations demonstrate the existence a new hydrogen clathrate hydrate can be synthesized at $1.2 \mathrm{GPa}$ and $298 \mathrm{~K}$ via pressure manipulation. The composition of this compound is $\left(\mathrm{H}_{2} \mathrm{O}\right)_{6} \mathrm{H}_{2}$, the same as of the familiar $C_{1}$ clathrate, while the structure is similar to $C_{1}$ clathrate and ice II but proton disordered. This is remarkable given that a disordered version of ice II is not known. These findings shed new light on the phase diagram of water and clathrates. Future neutron singlecrystal diffraction investigation would be instrumental in the confirmation of these results.

This work was supported by the National Natural Science Foundation of China (Grants No. 11504382, No. 21473211, No. 11674330, No. 51672279, No. 11874361, No. 117743354, and No. 51727806), the CASHIPS Director's Fund (Grant No. YZJJ201705), the Chinese Academy of Science (Grants No. YZ201524 and No. YZJJ2020QN22), and a Science Challenge Project No. TZ201601. Parts of this work were performed at GeoSoilEnviroCARS (The University of Chicago, Sector 13), Advanced Photon Source (APS), Argonne National Laboratory. GeoSoilEnviroCARS is supported by the National Science Foundation-Earth Sciences (EAR1634415) and Department of Energy-GeoSciences (DEFG02-94ER14466). The Advanced Photon Source is a U.S. Department of Energy (DOE) Office of Science User Facility operated for the DOE Office of Science by Argonne National Laboratory under Contract No. DEAC02-06CH11357. Work of V.R. was supported by A. R. O.'s Grant No. 2711.2020.2 to leading scientific schools from Russian Ministry of Science and Higher Education. Work of A. R. O is funded by Russian Science 
Foundation (Grant No. 19-72-30043). A. F. G. acknowledges support of the Army Research Office (Grants No. 56122-CH-H and No. 71650-CH W911NF-19-20172) and Carnegie Institution of Washington.

*Present address: Applied Physics Department, Soreq Nuclear Research Center (NRC), Yavne 81800, Israel.

[1] T. Bartels-Rausch, V. Bergeron, J. H. E. Cartwright, R. Escribano, J. L. Finney, H. Grothe, P. J. Gutiérrez, J. Haapala, W. F. Kuhs, J. B. C. Pettersson, S. D. Price, C. I. Sainz-Díaz, D. J. Stokes, G. Strazzulla, E. S. Thomson, H. Trinks, and N. Uras-Aytemiz, Rev. Mod. Phys. 84, 885 (2012).

[2] C. G. Salzmann, J. Chem. Phys. 150, 060901 (2019).

[3] J.E. D. Sloan and C.A. Koh, Chemical Industries, 3rd ed.(CRC Press, Boca Raton, FL, 2007).

[4] J. I. Lunine and D. J. Stevenson, Icarus 70, 61 (1987).

[5] W. L. Mao, H. K. Mao, A. F. Goncharov, V. V. Struzhkin, Q. Z. Guo, J. Z. Hu, J. F. Shu, R. J. Hemley, M. Somayazulu, and Y. S. Zhao, Science 297, 2247 (2002).

[6] Y. A. Dyadin, E. Y. Aladko, and E. G. Larionov, Mendeleev Commun. 7, 34 (1997).

[7] K. A. Lokshin, Y. Zhao, D. He, W. L. Mao, H. K. Mao, R. J. Hemley, M. V. Lobanov, and M. Greenblatt, Phys. Rev. Lett. 93, 125503 (2004).

[8] V. S. Efimchenko, M. A. Kuzovnikov, V. K. Fedotov, M. K. Sakharov, S. V. Simonov, and M. Tkacz, J. Alloys Compd. 509, S860 (2011).

[9] T. A. Strobel, M. Somayazulu, and R. J. Hemley, J. Phys. Chem. C 115, 4898 (2011).

[10] T. A. Strobel, M. Somayazulu, S. V. Sinogeikin, P. Dera, and R. J. Hemley, J. Am. Chem. Soc. 138, 13786 (2016).

[11] L. del Rosso, M. Celli, and L. Ulivi, Nat. Commun. 7, 13394 (2016).

[12] W. L. Vos, L. W. Finger, R. J. Hemley, and H. K. Mao, Phys. Rev. Lett. 71, 3150 (1993).

[13] D. Londono, W. F. Kuhs, and J. L. Finney, Nature (London) 332, 141 (1988).

[14] B. Kamb, in In Physics and Chemistry of Ice, edited by E. J. Whalley, S. J., and L. W. Gold, Eds. (Royal Society of Canada, Canada, 1973), pp. 28-41.

[15] T. Nakamura, M. Matsumoto, T. Yagasaki, and H. Tanaka, J. Phys. Chem. B 120, 1843 (2016).

[16] S.-i. Machida, H. Hirai, T. Kawamura, Y. Yamamoto, and T. Yagi, J. Chem. Phys. 129, 224505 (2008).

[17] G. R. Qian, A. O. Lyakhov, Q. Zhu, A. R. Oganov, and X. Dong, Sci Rep 4, 5606 (2014).

[18] C. G. Salzmann, P. G. Radaelli, E. Mayer, and J. L. Finney, Phys. Rev. Lett. 103, 105701 (2009).

[19] V. Diatschenko, C. W. Chu, D. H. Liebenberg, D. A. Young, M. Ross, and R. L. Mills, Phys. Rev. B 32, 381 (1985).
[20] See Supplemental Material at http://link.aps.org/supplemental/ 10.1103/PhysRevLett.125.255702 for Materials and Methods, Figs. S1-S8, Tables S1-S3, and bibliography, which includes Refs. [21-38].

[21] D. D. Ragan, R. Gustavsen, and D. Schiferl, J. Appl. Phys. 72, 5539 (1992).

[22] A. R. Oganov and C. W. Glass, J. Chem. Phys. 124, 244704 (2006).

[23] A. R. Oganov, A. O. Lyakhov, and M. Valle, Accounts Chem. Res. 44 (3), 227 (2011).

[24] A. O. Lyakhov, A. R. Oganov, H. T. Stokes, and Q. Zhu, Comput. Phys. Commun. 184, 1172 (2013).

[25] Q. Zhu, A. R. Oganov, C. W. Glass, and H. T. Stokes, Acta Crystallogr. Sect. B 68, 215 (2012).

[26] G. Kresse and J. Furthmüller, Phys. Rev. B 54, 11169 (1996).

[27] G. Kresse and D. Joubert, Phys. Rev. B 59, 1758 (1999).

[28] A. Tkatchenko, R. A. DiStasio, R. Car, and M. Scheffler, Phys. Rev. Lett. 108, 236402 (2012).

[29] A. Ambrosetti, A. M. Reilly, R. A. DiStasio, and A. Tkatchenko, J. Chem. Phys. 140, 18A508 (2014).

[30] D. Porezag and M. R. Pederson, Phys. Rev. B 54, 7830 (1996).

[31] A. Fonari and S. Stauffer, vasp_raman.py, https://github .com/raman-sc/VASP/, 2013.

[32] M. Dion, H. Rydberg, E. Schröder, D. C. Langreth, and B. I. Lundqvist, Phys. Rev. Lett. 92, 246401 (2004).

[33] J. Klimeš, D. R. Bowler, and A. Michaelides, J. Phys. Condens. Matter 22, 022201 (2009).

[34] J. Klimeš, D. R. Bowler, and A. Michaelides, Phys. Rev. B 83, 195131 (2011).

[35] L. Pauling, General Chemistry. (W.H. Freeman and Co., San Francisco 1970).

[36] R. E. Gagnon, H. Kiefte, M. J. Clouter, and E. Whalley, J. Chem. Phys. 92, 1909 (1990).

[37] L. Bezacier, B. Journaux, J. P. Perrillat, H. Cardon, M. Hanfland, and I. Daniel, J. Chem. Phys. 141, 104505 (2014).

[38] P. Loubeyre, R. LeToullec, D. Hausermann, M. Hanfland, R. J. Hemley, H. K. Mao, and L. W. Finger, Nature (London) 383, 702 (1996).

[39] Rigaku, CrysalisPro Software system (Rigaku Corporation, Oxford, UK 2017).

[40] O. V. Dolomanov, L. J. Bourhis, R. J. Gildea, J. A. K. Howard, and H. Puschmann, J. Appl. Crystallogr. 42, 339 (2009).

[41] G. Sheldrick, Acta Crystallogr. Sect. A 64, 112 (2008).

[42] V. Petricek, M. Dusek, and L. Palatinus, Z. Kristallogr. 229, 345 (2014).

[43] W. C. Hamilton, Acta Crystallogr. 18, 502 (1965). 